\title{
TEMA -2018: La hipertensión arterial supina en pacientes con desórdenes autonómicos: revisión anatómica y concep- tual
}
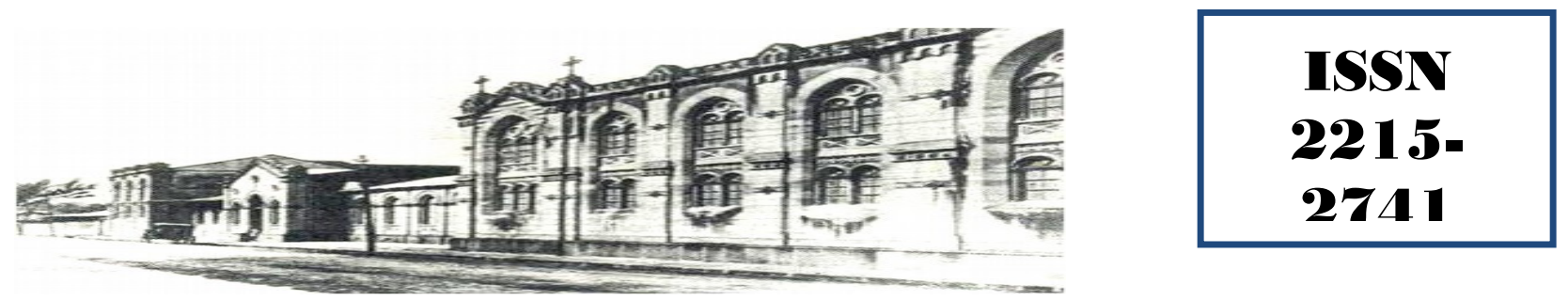

Haspital San quan de Dias, San Gasé, Casta Rica. Fundada en 1845

Recibido:

$31 / 01 / 2018$

Aceptado:

$15 / 03 / 2018$

Carlos Mario Sequeira Quesada ${ }^{1}$

Daniel Andrés Casares Fallas ${ }^{2}$

${ }^{1}$ Medico Cirujano General. Docente de la Escuela de Medicina y Educación Física en la Universidad de Costa Rica. carlossq24@hotmail.com

${ }^{2}$ Residente posgrado de Medicina Interna dcasaresf@hotmail.com

\section{RESUMEN}

El sistema nervioso está dividido en un sistema nervioso central, el sistema nervioso periférico y el sistema nervioso autonómico. Este sistema autónomo cumple funciones claves en la homeostasis, la adaptación y otras múltiples funciones autoregulatorias. El sistema nervioso autonómico a su vez se clasifica en una división simpática y otra parasimpática, ambas conformadas por una neurona pregangliónica y una posgangliónica. Centros a nivel central regulan las dos divisiones. Los trastornos del sistema nervioso autonómico se pueden manifestar de múltiples maneras, y se clasifican respectivamente en estructurales y funcionales según tengan o no lesiones anatómicamente reconocibles. La hipertensión arterial supina se caracteriza por una presión arterial elevada en dicha posición y si bien es cierto no se ha logrado documentar un daño a órgano blanco de la misma severidad como en la hipertensión arterial crónica, su importancia radica en que es común en pacientes con desórdenes autonómicos y aún más si estos reciben tratamiento por hipotensión ortostática. El tratamiento de la hipertensión arterial supina radica en una serie de medidas no farmacológicas y farmacológicas que tienen el objetivo de sopesar el riesgo de caídas, síncopes y tener tolerancia ortostática contra el posible daño a órgano blanco producido por los niveles elevados de la presión arterial.

\section{PALABRAS CLAVE}

Autonómico. SNA. Disautonomía. Hipertensión arterial. Hipertensión supina.

\section{ABSTRACT}

The nervous system is divided in a central nervous system, a peripheral nervous system and an autonomic nervous system. The autonomous system has functions related to homeostasis, adaptation and other multiple autoregulatory tasks. The 
autonomic nervous system is divided in a sympathic and a parasympathic branch, both conformed by a preganglionic and a postganglionic neuron and both regulated upwards by central centers. The patologies affecting this system can manifest themselves in a myriad of ways and can be classified in structural and functional disorders depending on whether or not they have a defined anatomic injury. Supine hypertension is characterized by an elevated blood pressure in the aforementioned position and eventhough unlike chronic elevated blood pressure end organ damage has not been fully documented, its importance lies on its high incidence in dysautonomic patients and moreso if they receive medications for ortosthatic hypotension. Treatment for supine hypertension consists in a series of non-medical and pharmacologic measures with the goal of balancing the risk of falls, syncope, and orthostatic intolerance against the possible end organ damage produced by the elevated blood pressure levels.

\section{KEY WORDS}

Autonomic. ANS. Dysautonomia. Arterial hypertension. Supine hypertension.

\section{INTRODUCCIÓN}

El cerebro humano es el órgano más complejo del cuerpo. Está encargado de vastísimas funciones como mediar comportamientos que van desde movimientos simples hasta la percepción sensorial, la memoria y el aprendizaje. Es el llamado órgano de la mente, con muchísimas funciones aún no completamente entendidas. De hecho, quizás de las funciones más llamativas para el ser humano como resulta la función del pensamiento, persiste como una parcial interrogante hasta la fecha. El cerebro en toda su complejidad no es sino un componente de un sistema mucho más amplio, que conceptualizamos como sistema nervioso ${ }^{1}$.

El sistema nervioso en sí está subdividido de una manera hasta cierto punto arbitraria. Todos los elementos de él trabajan integradamente en cercanía, en una forma aparentemente sin límites claros. No obstante, la separación tradicional en subdivisiones proporciona una fragmentación útil para poder valorar todas las conexiones y funcio- nes de los elementos dentro de él, y resulta importante en ese contexto. Es entonces que se procede a la separación del sistema nervioso en el llamado sistema nervioso central, el cual abarca el encéfalo (entiéndase cerebro, cerebelo y tallo cerebral) y el cordón espinal. Subsecuentemente se tiene el sistema nervioso periférico, dentro del cual se aglomeran todos los componentes extradurales, ennumerándose nervios espinales, pares craneales (a excepción del nervio olfatorio y óptico los cuales por su origen embriológico se consideran parte del sistema nervioso central) y junto a ellos los receptores periféricos y ganglios de la raíz dorsal que por función trabajan cercanamente entre sí. Ciertos autores incluyen como parte de este sistema inclusive a la placa motora (axón de neurona motora y todas las fibras musculares que esta inerva). Por último y como tercera subdivisión, se erige el llamado sistema nervioso autonómico ${ }^{1}$.

El sistema nervioso autonómico es el componente crítico en una red central involucrada en la homeostasis y la adaptación. Cumple con múltiples funciones como la regulación de la presión arterial y el flujo sanguíneo regional en respuesta a las demandas metabólicas de diversos tejidos, la termorregulación, motilidad y secreción del tracto respiratorio y gastrointestinal, la micción y la función sexual. El sistema nervioso autonómico inerva todos los órganos en el cuerpo. Como será profundizado en la sección de revisión anatómica, a pesar de que se subclasifica por aparte, el sistema nervioso autónomo está conformado por estructuras o partes de tanto el sistema nervioso central como del periférico.

La diseminación de su alcance causa que las manifestaciones de un mal funcionamiento de este sistema, evento conocido con el nombre de disautonomías, sean múltiples y extensas, requiriendo por ende una amplia valoración clínica para dirigir la subsecuente evaluación de laboratorio. El abordaje clínico depende principalmente de una historia clínica detallada y un examen físico preciso, seguido de pruebas de laboratorio de naturaleza tanto general como específicamente dirigidas.

Lo anterior guía al médico tratante a realizar una síntesis de la información recolectada, fundamentando subsecuentemente una impresión diagnóstica y una formulación de un plan de manejo individualizado según el paciente y su presenta- 
ción clínica. Las metas de la evaluación clínica son identificar la presencia, distribución y patrón temporal de disfunción autonómica, así como especificar los componentes de dicho sistema nervioso que se encuentren comprometidos. Es de suma importancia a su vez la detección de desórdenes que, si bien pudiesen ser serios, resulten tratables $^{2,3}$.

Los síntomas de compromiso autonómico abarcan diferentes estructuras tanto a nivel periférico como central, generando disfunción de diferentes maneras en órganos y sistemas como los involucrados en visión, cardiovascular, pulmonar, gastrointestinal, el tracto urinario, la función sexual, la generación de hemocomponentes y mantenimiento de una sudoración apropiada. Las patologías causando esta disfunción son de distintas índoles, oscilando desde lo funcional hasta enfermedades con neurodegeneración, con cursos temporales agudo, subagudo y/o crónico. Los pacientes con desórdenes autonómicos tienen hallazgos asociados a sus patologías de fondo tanto autonómicas como no autonómicas.

Dentro de estas manifestaciones se encuentra la hipertensión supina. A lo largo de este artículo se realizará una revisión anatómica detallando los diferentes componentes anatómicos conocidos del sistema nervioso autonómico. Posteriormente se trasladará estos conocimientos a una forma de clasificación de las diferentes patologías que comprometen dicho sistema, para finalmente definir el concepto de hipertensión supina y las diferentes posibilidades terapéuticas con las que cuenta el médico para abordarla ${ }^{4}$.

\section{DISCUSIÓN}

\section{Revisión Anatómica}

El sistema nervioso autonómico o autoregulador está distribuido en los tejidos y órganos periféricos en la forma de ganglios autonómicos, mientras que los centros controladores del sistema se localizan a nivel central, en estructuras como el hipotálamo y el tallo cerebral. Por lo anterior se dice el sistema nervioso autónomo acarrea señales desde el cerebro hacia la periferia. A modo de simplificación, se establece que el sistema nervioso autonómico está compuesto por dos neuronas.
La primera neurona está ubicada a nivel central, ya sea en la columna visceral aferente general del cerebro o en el cordón espinal y es conocida como neurona pregangliónica. Los previamente mencionados centros controladores en el sistema nervioso central envían fibras a hacer sinapsis con dichas neuronas pregangliónicas. Estas fibras no se incluyen dentro la esquematización de dos neuronas mencionada sin embargo ejemplifica la vasta regulación a diferentes niveles que existe en el sistema nervioso central.

Las neuronas pregangliónicas envían pequeños axones mielinizados a los ganglios autonómicos localizados en la periferia, y también al sistema nervioso entérico, subdivisión del sistema autonómico. Es en estos ganglios donde ocurre la primera sinapsis. Las neuronas nacidas en dichos ganglios emiten axones no mielinizados, los cuales se conocen como la segunda neurona del sistema o neuronas postgangliónicas y estas inervan a los distintos órganos blanco ${ }^{3,5}$.

Tanto anatómica como funcionalmente el sistema autonómico está compuesto por dos divisiones, las denominadas división simpática y la parasimpática. A pesar de lo anterior el sistema autonómico se encuentra completamente integrado como un todo con la actividad motora y el sistema neuroendocrino. Si bien es cierto la mayoría de las funciones de este sistema se llevan a cabo en un nivel involuntario o inconsciente, no está exento de modulación cortical y subcortical ${ }^{3,5}$.

Previo a profundizar en dichas subdivisiones, se debe hacer mención a los citados componentes reguladores de las redes a nivel central. A este nivel son importante diferentes estructuras telencefálicas como la corteza insular, la corteza cingulada anterior y la amígdala. También se encuentran relacionadas estructuras diencefálicas como el hipotálamo y estructuras en el tallo cerebral como lo son la sustancia gris periacueductal, el núcleo del tracto solitario, la formación reticular ventrolateral medular y el rafé medular.

Todas estas estructuras como fue ya mencionado ejercen un control superior al esquema de dos neuronas utilizado para simplificar el sistema autonómico, y están íntimamente conectados entre sí para cumplir un rol crítico en la integración de sensaciones corporales, respuestas endocrinas, emoción, toma de decisiones y respuestas a estrés externo ${ }^{3,6,7,8}$. 
El sistema simpático tiene sus neuronas pregangliónicas a nivel de cordón espinal, específicamente en el núcleo intermediolateral entre los niveles T1 y L2. Estas neuronas están organizadas en agrupaciones o cúmulos neuronales, sin embargo su distribución no sigue los patrones en dermatomas sí seguidos por los nervios somáticos.

Dentro de este sistema simpático la inervación de la cabeza recae en los niveles $\mathrm{T} 1$ a $\mathrm{T} 3$, de $\mathrm{T} 1$ a T6 las extremidades superiores y las correspondientes vísceras torácicas. Los niveles T5 a T11 otorgan la inervación de las vísceras abdominales mientras que de T11 a L2 las extremidades inferiores y los órganos pélvicos y perineales. Los axones pregangliónicos hacen su salida a través de las raíces ventrales y por medio de los llamados ramos comunicantes blancos (por su mielinización) corriendo como parte de los nervios espinales correspondientes llegan a la cadena simpática paravertebral.

La mayoría de estas fibras pregangliónicas corren rostral o caudalmente a lo largo de la cadena simpatética y sinapsan no en uno sino en varios de los ganglios que la componen. Las fibras restantes que no hacen este recorrido no hacen conexión en esta cadena, sino que forman los nervios esplácnicos e inervan los ganglios prevertebrales y la médula suprarrenal. Los ganglios paravertebrales son principalmente estaciones de relevo para las aferencias pregangliónicas. Inervan a todos los órganos y tejidos del cuerpo a excepción de aquellos en abdomen, pelvis y periné.

Por otro lado, los ganglios prevertebrales se localizan anterior a la aorta en cercana relación con las arterias celíaca y mesentérica. Estos ganglios son los encargados de proveer la inervación para los faltantes abdomen, pelis y periné. A diferencia de los paravertebrales, los ganglios prevertebrales no funcionan precisamente como estaciones de relevo, sino que en estos ganglios se integran inputs aferentes pregangliónicos con las aferencias periféricas desde los ganglios de la raíz dorsal y neuronas sensoriales del sistema nervioso entérico. Por ende, se conceptualiza los ganglios prevertebrales como el sitio de los reflejos gangliónicos y son independientes del sistema nervioso central y la modulación de sus centros citada previamente ${ }^{3,5}$.
Por su parte, el sistema parasimpático se origina de neuronas pregangliónicas localizadas en núcleos del tallo cerebral y cordón espinal en el segmento sacro. Los axones pregangliónicos parasimpáticos recorren una distancia mayor a sus contrapartes simpáticas. Eventualmente dichos axones alcanzan sus ganglios objetivo, los cuales normalmente se encuentran más cerca o inclusive dentro de los órganos blanco. Los núcleos parasimpatéticos pregangliónicos craneales se proyectan a través de los pares craneales oculomotor, facial, glosofaríngeo y vago.

Todos los anteriores cumplen con diversas funciones y alcanzan distintos objetivos, sin embargo, la mayoría del output del tallo cerebral se realiza a través del décimo par craneal o nervio vago. Este es particularmente importante debido a su inervación de estructuras como el corazón, tracto respiratorio y todo el tracto gastrointestinal a excepción del colon descendente y el recto.

El vago ejerce entonces efectos cardioinhibitorios, visceromotores y secretomotores. La segunda parte del sistema parasimpático es su componente sacro preganglionar, el cual nace del núcleo localizado en la sustancia gris lateral entre los segmentos espinales S2 y S3. Sus axones pasan por las raíces ventrales y se adjuntan a los nervios esplácnicos, los cuales a su vez se unen al plexo hipogástrico inferior e inervan el colon, vejiga y órganos sexuales. Las neuronas pregangliónicas sacras están involucradas en interacciones inhibitorias recíprocas con motoneuronas somáticas del núcleo de Onuf a través de interneuronas, las cuales inervan la uretra externa y el esfínter rectal, así como el piso pélvico a través del nervio pudendo ${ }^{3,5}$.

Por último, el sistema nervioso entérico, también parte del sistema nervioso autonómico, incluye varios tipos de neuronas sensoriales, interneuronas y neuronas de índole motora. Estas neuronas están localizadas dentro de las paredes intestinales en el plexo mientérico y submucoso. Ambos plexos forman circuitos reflejos locales que median la motilidad, secreción y el flujo sanguíneo a través de las asas intestinales.

Aunque el control de estos órganos es llevado a cabo a través de estos reflejos segmentarios, aferencias del nervio vagal y los ganglios prevertebrales pueden modular su actividad. Se puede también resaltar el componente aferente visceral 
del mismo, el cual informa al sistema nervioso central de eventos mecánicos y químicos en los órganos internos. Esta información es recibida para producir una sensación visceral consciente incluyendo dolor e iniciar las respuestas reflejas viscerales. Se pueden separar los aferentes viscerales en espinales y de tallo cerebral. Todos los nervios aferentes del tallo relevan en el importante núcleo del tracto solitario ${ }^{3,5}$.

Además de centrarnos en una revisión anatómica y estructural del sistema nervioso autonómico, conviene hacer una rápida mención al sistema de neurotransmisores utilizados en las diferentes sinapsis y puntos de relevo mencionados. El transmisor principal a nivel pregangliónico en tanto la subdivisión simpática como parasimpática es la acetilcolina, actuando en receptores de tipo nicotínico.

La acetilcolina es también el neurotransmisor primario de todas las neuronas postgangliónicas parasimpáticas, así como de aquellas neuronas simpáticas que inervan las glándulas sudoríparas. La acción en estos sitios la ejercen a través de receptores muscarínicos. Por último, el neurotransmisor de las restantes neuronas postgangliónicas simpáticas es la norepinefrina, la cual actúa en alguno de la gran variedad de receptores adrenérgicos existentes ${ }^{3,5}$.

\section{Clasificación trastornos autonómicos}

$\mathrm{Al}$ momento de tomar la historia médica enfocada a problemas de índole autonómico, el examinador debe tener en mente los principales componentes del sistema autonómico previamente mencionados, como lo son los componentes simpáticos noradrenérgicos, simpáticos colinérgicos, parasimpáticos colinérgicos, adrenomedulares y disfunciones entéricas, debido a que el compromiso de cada uno de ellos se manifiesta de diferentes maneras.

Las características, el inicio, evolución, factores tanto agravantes como que aminoren la severidad de lo síntomas y posible relación de los mismos con las comidas, actividades de la vida diaria, temperatura ambiental y tiempo del día son importantes. Una revisión completa de los sistemas debe ser realizada, con especial atención a síntomas autonómicos que sean difíciles de evaluar con pruebas de laboratorio. En adición a obtener una historia neurológica, así como documentar quejas como mareo posprandial y documentar la función vasomotora, sudomotora, pupilomotora, vesical, intestinal, de sueño, respiratoria y sexual.

Es igual de importante registrar una lista completa de todas las medicaciones prescritas u obtenidas por otros medios, remedios naturales y suplementos dietéticos, esto último no solo porque pueden afectar la función autonómica sino porque también pueden interactuar para producir eventos adversos serios y no esperados ${ }^{3}$.

Desde una perspectiva neurológica, los desórdenes autonómicos son entendidos de una mejor manera utilizando un esquema conceptual similar al utilizado en desórdenes de otros sistemas, como por ejemplo el gastrointestinal. A priori, un desorden puede ser entendido como funcional o estructural.

Entonces, en el mundo de patologías autonómicas, aquellos desórdenes entendidos como estructurales, a veces también referidos como fallas autonómicas, son definidos por tener una anormalidad patológica demostrable que interfiere directamente con las vías neurales autonómicas previamente detalladas. Ejemplos de estas patologías son la atrofia sistémica múltiple, el mal de Parkinson o la neuropatía diabética autonómica.

En contraparte, los desórdenes funcionales no tienen un sustrato anatómico demostrable, y se definen primariamente por la sintomatología que presenta el paciente y frecuente constituye un síndrome. Ejemplos de este grupo son el síndrome de taquicardia postural, el síndrome de dolor regional complejo y el síndrome de intestino irritable. Evidentemente, los desórdenes pueden pasar del cajón de funcional a estructural a lo largo del tiempo conforme nuestro conocimiento de las etiologías evolucione y avance ${ }^{4}$.

Ya que por definición los desórdenes estructurales tienen un punto de origen anatómico conocido, estos se pueden dividir en dos de acuerdo, originando entonces desórdenes periféricos y patologías centrales.

Esta división no es fácilmente trasladable a los desórdenes funcionales. A su vez, los desórdenes periféricos se pueden subseparar según sea las fibras aferentes o las eferentes las más comprometidas. Debe ponderarse que pocos de los desórdenes estructurales son completamente puros en el sentido de solo afectar localizaciones centrales o 
periféricas. Por ejemplo, la diabetes puede involucrar tanto nervios periféricos como las vías centrales. Las patologías estructurales y funcionales difieren unas de las otras en múltiples sentidos.

Los entes estructurales, a pesar de generar cambios patológicos extensos en el sistema nervioso, tienden a propiciar pocos síntomas. En contraste, los pacientes con desórdenes funcionales presentan grandes y numerosas quejas muchas veces desproporcionadas con su supuesta falta de afectación anatómica. En general, todos estos desórdenes se conceptualizan actualmente como multiorgánicos en vez de males de un solo sitio. Otra diferencia entre las patologías estructurales y funcionales recae en el pronóstico de cada una, esto pues los estructurales tienen un desenlace más sombrío ${ }^{4}$.

Diferencias entre la hipertensión arterial supina y la hipertensión arterial crónica

Como fue mencionado, además de las diferentes manifestaciones tradicionales propios de cada entidad estructural o funcional, estos pacientes tienen síntomas autonómicos y no autonómicos. Dentro de estas manifestaciones autonómicas se encuentran las diferentes alteraciones en la presión arterial, no entendiéndose por esto únicamente una elevación en las cifras arteriales, sino todo el espectro de afectación que puede existir.

Este espectro incluye la hipertensión arterial crónica, la hipotensión ortostática y la hipertensión arterial supina, aunque en este documento nos centraremos únicamente en las formas de hipertensión mencionadas. La hipertensión arterial supina se comprende como aquella que aparece cuando se ha estado en posición supina por 15 minutos, a pesar de que en algunos estudios no describen un tiempo límite particular para su definición si no solamente la existencia de la posición específica mencionada.

No existen definiciones formales referentes al nivel de tensión arterial a partir del cual conceptualizar la hipertensión arterial supina, sin embargo se han propuesto niveles de 150/90. Por su parte, la hipertensión arterial crónica en la esfera clínica se puede definir como el nivel de presión arterial en el cual el tratamiento que se emprenda disminuye las cifras de morbilidad y mortalidad, siendo este nivel el promedio de dos o más lecturas de presión arterial (sujeto sedente) durante dos o más visitas extrahospitalarias al menos separadas de un mes.

En general en las clasificaciones de hipertensión arterial el valor inicial a partir del cual se define es $\geq 140 / 90 \mathrm{mmHg}$. Recientemente esta definición ha sufrido un cambio, indicándose que pacientes con niveles mayores a 130/80 deben ser entendidos ya como personas con hipertensión arterial. Citamos sin embargo la previa definición debido al escepticismo con el que ha sido recibida las nuevas cifras recomendadas $9,10,11,12,13,14,15,16,17,18$.

Cómo se puede ver, las diferencias de ambos conceptos son evidentes desde la misma definición, pero clínicamente también es relevante tener presente estos diagnósticos debido al efecto a largo plazo de cada una sobre los órganos y sistemas corporales. A diferencia de la hipertensión arterial supina, como se detallará a continuación, el daño a órgano blanco si está mejor definido en sitios como corazón, apoplejía, riñón y enfermedad arterial periférica por citar algunos sitios.

En cuanto al daño a órgano blanco en la hipertensión arterial supina se ha reportado que efectivamente si existe compromiso, específicamente a nivel cardíaco en la forma de miocardiopatía hipertrófica y con un mayor compromiso de ventrículo derecho, sin embargo, otros sitios de afección común en la forma crónica como son la enfermedad cerebrovascular y la nefropatía no han sido estudiados a fondo. Se hipotetiza que el agrandamiento de cámaras es debido al mantenimiento de presiones arteriales elevadas durante los períodos de sueño ${ }^{10,11,13,15,19}$.

Sobre la fisiopatología de la hipertensión arterial crónica, una revisión a fondo de la misma resulta fuera de los objetivos de esta revisión y con la suficiente extensión para meritar un documento propio, sin embargo, se habla de una hipertensión arterial esencial o primaria, generando aproximadamente un $90 \%$ de los casos, y una amalgama de etiologías, si bien es cierto no tan frecuentes, que propician elevación de las tensiones arteriales de una manera secundaria. Referente a la mencionada hipertensión arterial esencial se han establecido mecanismos relacionados con el volumen intravascular, el sistema nervioso autónomo, una hiperactividad a nivel sistema renina an- 
giotensina aldosterona y alteraciones vascular, como cambios en el grosor de las arterias renales 13 .

Epidemiológicamente la hipertensión arterial supina es más frecuente en pacientes con desórdenes autonómicos, en especial si estos son tratados por hipotensión ortostática. Muchos pacientes con este desorden presentan hipertensión supina inclusive antes de recibir el tratamiento dirigido a mejorar la hipotensión ortostática, y por ende esta se ve exacerbada cuando se instaura la fludrocortisona y los agentes presores. Según la enfermedad de base autonómica se han estudiado diversos mecanismos que expliquen la hipertensión supina mas no se ha logrado unificar una única vía en común para todas. En pacientes con falla autonómica pura se ha hipotetizado sobre una menor ganancia del baroreflejo y del reflejo cardiovagal.

Además, los niveles de norepinefrina han sido menores en pacientes con hipertensión arterial supina en comparación con los que no la poseen, sugiriendo mecanismos presores independientes al sistema autónomo en enfermedades como el Parkinson y enfermedad multisistémica atrófica. En esta última también hay un aumento en el tono simpático neurovascular. Existen ensayos en los cuales se ha documentado que el bloqueo gangliónico reduce la presión supina, implicando entonces que una eferencia simpática inapropiada podría ser clave en la fisiopatología de esta entidad $^{4,11,15}$.

La magnitud de la severidad de la hipertensión arterial supina en pacientes con hipertensión arterial crónica esencial que no tienen hipotensión ortostática fue similar a los pacientes que tienen hipertensión arterial supina con hipotensión ortostática, por lo que se confirma que la magnitud de las presiones supinas no guarda relación con una u otra enfermedad. Pese a lo anterior si se debe reforzar el concepto de la íntima relación entre la hipotensión ortostática neurogénica, su tratamiento y la hipertensión arterial supina ${ }^{11,20}$.

La hipotensión arterial ortostática fue previamente mencionada. Este concepto no será profundizado en este documento, sin embargo es importante tenerlo presente no solo pues como fue mencionado los pacientes que se encuentran en tratamiento por ella aumentan su riesgo de tener hipertensión supina, sino porque en los primeros abordajes del paciente con disautonomía es importante reconocer la diferencia fundamental entre el tratamiento de la hipertensión en la práctica clínica contra el tratamiento de la hipotensión supina en el paciente autonómico.

Cuando los médicos tratan hipertensión arterial crónica, el objetivo del mismo consiste en evitar eventos adversos letales, mientras que cuando se trata la hipotensión arterial crónica lo que se busca es mejorar la capacidad funcional del paciente y aminorar sus síntomas. Es dentro de estos extremos plantados por estos dos desórdenes que se encuentra a terapéutica de la hipertensión arterial supina ${ }^{4}$.

\section{Tratamiento de la Hipertensión Arte-} rial Supina

Como ha sido previamente establecido, la motivación de tratar la hipertensión arterial supina es mejorar la sintomatología del paciente. Aunado a esto, y ponderando que muchas veces esta puede ser de una considerable gravedad, existe además el riesgo a futuro de daño órgano blanco el cual poco a poco vamos dilucidando y ampliando nuestro conocimiento sobre él.

Tratar la hipertensión arterial supina es también importante para prevenir las complicaciones que se pueden generar por elevaciones agudas de los niveles tensionales, como lo puede ser una hemorragia cerebral. El abordaje terapéutico a disposición se puede separar en recursos no farmacológicos y aquellos basados en el uso de fármacos.

Es relevante reconocer la muy frecuente coexistencia entre hipotensión ortostática y la hipertensión arterial supina. Los expertos recomiendan abordar de manera separada las dos. Entre las medidas no farmacológicas resalta el evitar acostarse durante el día. Además, cuando eventualmente sí se va adoptar la posición supina es recomendable inclinar la cabecera de la cama 10-20 grados para estar semisentados, lo cual también disminuye la diuresis nocturna que produce la hipertensión arterial supina y su depleción de volumen.

Este último aspecto es importante a la hora de monitorizar a los pacientes, debido a que su presencia fisiopatológicamente puede generar síntomas de intolerancia al ortostatismo y acarrear la 
prescripción de tratamiento para la hipotensión ortostática, lo cual como ya fue mencionado en una naturaleza dosis dependiente produce como efecto secundario hipertensión arterial supina, hasta en un $50 \%$ en algunos estudios). Finalmente, podría ser necesario tener que aceptar cierto nivel de hipertensión supina con el fin de mantener la tolerancia ortostática, ya que la meta del tratamiento es disminuir los síntomas, deambular y no precisamente ser normotenso, además no se han precisado niveles de presión arterial específicos meta ${ }^{4,9,10,11,21,22}$.

Lo anterior entonces plantea la necesidad al momento de abordar un paciente con autonomía avanzada o tal vez de corta duración, pero con importante severidad de intentar balancear la hipotensión ortostática con la hipertensión supina. Resulta importante remarcar que el objetivo en el tratamiento de la hipertensión supina nunca debe ser lograr un control estricto de los niveles tensionales, sino mantener al paciente en un rango aceptable que prevenga tanto la molesta sintomatología como que además disminuya los riesgos asociados a ella.

Existen corrientes terapéuticas que recomiendan centrarse únicamente en abordar la hipotensión ortostática, limitándose a tratar la hipertensión arterial supina cuando esta llegue a niveles de 170/95. Además de las opciones terapéuticas no basadas en el uso de drogas, farmacológicamente el uso de antihipertensivos de acción corta titulados (por ejemplo, captopril e hidralazina) pueden ser utilizados en pacientes con hipertensión arterial supina sostenida, aunque siempre hay que valorar el riesgo beneficio en cuanto a caídas y síncopes, en especial en pacientes con fallas autonómicas. Otras opciones antihipertensivas son el propanolol, la clonidina y la nifedipina ${ }^{4,9,22,23}$.

Los parches de nitroglicerina usados en posición supina y retirados antes de ponerse de pie han sido reportados en 2 estudios con resultados beneficiosos. Otra opción es la piridostigmina, un inhibidor de la acetilcolinesterasa usada en pacientes con hipotensión ortostática neurogénica y es el que tiene menor elevación de la presión arterial supina en comparación con otros vasopresores. Inclusive se ha teorizado si dosis pequeñas de vasopresores combinados con piridostigmina tendrían el mejor efecto en el tratamiento de la hipotensión ortostática sin aumentar la presión arterial supina $^{4,9,12,24,25}$.
Recursos quirúrgicos como el previamente mencionado bloqueo de ganglios autonómicos se erigen como opciones, sin embargo, la radicalidad de estos abordajes los dejan como posibilidades poco prácticas en el manejo del día a día. Además, otorga una resolución crónica a un problema cuya paliación de momento ostenta una naturaleza e importancia más aguda o inmediata ${ }^{4,25}$.

Discutir metas realistas para la terapia de los pacientes es el objetivo central del abordaje por parte del médico, y debe hacer énfasis en el mensaje que un control perfecto de la presión arterial es poco probable, en cambio la meta para el tratamiento es asegurar su seguridad y prevenir síntomas serios. Realizar esta discusión en el plano de un abordaje multidisciplinario es importante para garantizar al paciente que los objetivos planteados son en verdad alcanzables. Proveer una perspectiva holística de la enfermedad y su tratamiento podría ultimadamente ayudar a los pacientes en la aceptación de su condición y podría potencialmente mejorar significativamente su calidad de vida) ${ }^{23}$.

\section{CONCLUSIONES}

La amplia extensión corporal del sistema nervioso autónomo y su cobertura de múltiples órganos y tejidos hacen que su disfunción se manifiesta en una gran cantidad de maneras. Las patologías que producen su disfuncionamiento se pueden clasificar en estructurales y funcionales, y las primeras a la vez subdividirse en centrales y periféricas según la ubicación precisa de la estructura correspondientemente lesionada.

Estas enfermedades abarcan síntomas tanto autonómicos como no autonómicos, dentro de los que se incluye la hipertensión arterial supina. Se han propuesto diferentes mecanismos fisiopatológicos de la hipertensión arterial supina, intentando correlacionarlo con enfermedades ligadas a desórdenes autonómicos sin haberse logrado todavía alcanzar un mecanismo único para todas las disautonomías. Se resalta sin embargo una disfunción del baroreflejo, reflejo cardiovagal y disfunciones generalizadas del tono simpático como puntos clave en la fisiopatología.

Aún faltan estudios que confirmen la magnitud y los niveles a los que se produce daño a los diferentes órganos blanco para que se logre definir 
una meta de tratamiento mucho más objetiva. $\mathrm{Al}$ no tener aún dicho objetivo, no se puede definir el impacto que tendrán las medidas farmacológicas y no farmacológicas sobre puntos duros como la mortalidad.

En la actualidad los objetivos terapéuticos se deben limitar a que el paciente tenga funcionalidad en cuanto a deambular y tolerancia ortostática contra un nivel de presión arterial que no siempre va a ser normal mas si prevenir molesta sintomatología aguda, así como complicaciones agudas de índole hemorrágica a nivel cerebral. En caso de fallar las opciones no farmacológicas se recurre a antihipertensivos de corta duración para lograr el rápido descenso de los niveles arteriales.

Este abordaje es favorecido cuando el paciente presenta presiones arteriales muy elevadas, aunque el nivel exacto para el cual el tratamiento se recomienda no está oficializado.

\section{BIBLIOGRAFÍA}

1. Boron W, Boulpaep E. Medical Physiology. Second edition Updated edition. Chapter 10: Organization of the Nervous System. Elsevier Saunders. 2012: 267-270.

2. Goldstein D, Low P. Clinical Evaluation of the Autonomic Nervous System. Continuum Lifelong Learning Neurol 2007;13(6):33-49.

3. Benarroch E. The Autonomic Nervous System: Basic Anatomy and Phisiology. Continuum Lifelong Learning Neurol 2007;13(6):13-32.

4. Daroff R, Jankovic J, Mazziotta J et al. Bradley's Neurology in Clinical Practice. Seventh Edition. Chapter 108: Disorders of the Autonomic Nervous System. Elsevier. 2016; 1867-1895.

5. Mtui E, Gruener G, Dockery P. Fitzgerald's Clinical Neuroanatomy and Neuroscience. $7^{\text {th }}$ edition. Chapter 13: Auto- nomic Nervous System. Elsevier 2016:136-150.

6. Benarroch E. The central autonomic network: functional organization, dysfunction, and perspective. Mayo Clin Proc 1993;68(10):988-1001.

7. Saper C. The central autonomic nervous system: conscious visceral perception and autonomic pattern generation. Annu Rev Neurosci 2002;25: 433-469.

8. Toni R, Malaguti A, Benfenati F, Martini L. The human hypothalamus:a morpho-functional perspective. J Endocrinol Invest 2004;27(6 suppl):73-94.

9. Fanciulli A, Wenning A, Gregor K. Multiple-System Atrophy. New England Journal of Medicine 2015: 249-263.

10. Freeman R. Neurogenic Orthostatic Hypotension. New England Journal of Medicine. 2008: 615-624.

11. Goldstein D, Pechnik S, Holmes C et al. Association between supine hypertension and orthostatic hypotension in autonomic failure. Hypertension 2003; 42: 136-42.

12. Jordan J, Shannon J, Pohar B et al. Contrasting effects of vasodilators on blood pressure and sodium balance in the hypertension of autonomic failure. J Am Soc Nephrol 1999;10-35.

13. Kotchen T. Vasculopatía hipertensiva. McGraw-Hill 2016.

14. Whelton P, Carey R, Aronow W et al. Guideline for the Prevention, Detection, Evaluation and Management of High Blood Pressure in Adults. Hypertension.2017; 1-481. 
15. Freeman R, Kaufmann H. Disorders of Orthostatic Tolerance- Orthostatic Hypotension, Postural Tachycardia Syndrome, and Syncope. Continuum Lifelong Learning Neurol 2007;13(6):5088.

16. Kanjwal K, George A, Figueredo VM, Grubb BP. Orthostatic hypotension: definition, diagnosis and management. $\mathrm{J}$ Cardiovasc Med (Hagerstown). 2015;16(2):75-81.

17. Naschitz JE, Slobodin G, Elias N, Rosner I. The patient with supine hypertension and orthostatic hypotension: a clinical dilemma. Postgrad Med J. 2006;82(966):246-253

18. Lamarre-Cliche M. Orthostatic hypotension and supine hypertension in the patient with autonomic failure. Can J Gen Intern Med. 2014;9(3):91-95.

19. Vagaonescu T, Saadia D, Tuhrim S et al. Hypertensive cardiovascular damage in patients with primary autonomic failure. Lancet 2000:725-726.

20. Kaufmann H, Goldstein D. Autonomic Failure in Neurodegenerative Disorders. Continuum Lifelong Learning Neurol 2007;13(6):111-142.

21. Singer W, Sandroni P, Opfer-Gehrking T et al. Pyridostigmine Treatment Trial in Neurogenic Orthostatic Hypotension. Arch Neurol. 2006; 63(4):513518.

22. Ahmed A, Grubb B, Zaman L et al. Syndrome of Supine Hypertension with Orthostatic Hypotension. A nightmare for Physicians. The Journal of Innovations in Cardiac Rhythm Management 2016; 7: 2285-2288.
23. Nwazue V, Raj S. Confounders of vasovagal syncope: orthostatic hypotension. Cardiol Clin 2013; 31:89.

24. Shannon J, Jordan J, Costa F et al. The hypertension of autonomic failure and its treatment. Hypertension 1997;30:1062.

25. Shannon J, Jordan J, Dietrich A et al. Sympathetically mediated hypertension in autonomic failure. Circulation 2000; 101 (23), 2710-2715.

\section{CONFLICTO DE INTERÉS Y/O AGRADECIMIENTOS}

Los autores declaran que no existió ningún conflicto de interés en el presente reporte. 